\title{
Supermanifolds Theory and Applications
}


This page intentionally left blank 


\title{
Supermanifolds Theory and Applications
}

\author{
Alice Rogers \\ King's College London
}


Published by

World Scientific Publishing Co. Pte. Ltd.

5 Toh Tuck Link, Singapore 596224

USA office: 27 Warren Street, Suite 401-402, Hackensack, NJ 07601

UK office: 57 Shelton Street, Covent Garden, London WC2H 9HE

\section{British Library Cataloguing-in-Publication Data}

A catalogue record for this book is available from the British Library.

\section{SUPERMANIFOLDS: THEORY AND APPLICATIONS}

Copyright (C) 2007 by World Scientific Publishing Co. Pte. Ltd.

All rights reserved. This book, or parts thereof, may not be reproduced in any form or by any means, electronic or mechanical, including photocopying, recording or any information storage and retrieval system now known or to be invented, without written permission from the Publisher.

For photocopying of material in this volume, please pay a copying fee through the Copyright Clearance Center, Inc., 222 Rosewood Drive, Danvers, MA 01923, USA. In this case permission to photocopy is not required from the publisher.

ISBN-13 978-981-02-1228-5

ISBN-10 981-02-1228-3

Printed in Singapore. 
to Richard 


\section{Preface}

The subject of supermanifolds has to some extent 'just growed' like Topsy, as the idea of adjoining anticommuting variables to conventional commuting variables proved useful in a variety of contexts. This has led to a bewildering variety of approaches which has often obscured the underlying unity of the ideas. Supermanifolds have been with me through much of this period of growth, and now in writing this book I intend not a formal mathematical treatise, but rather a working man or woman's guide to the geometry and analysis of supermanifolds, together with applications of the theory. Supermanifolds continue to find new uses, the underlying ideas have proved robust, powerful and adaptable. My aim is to provide a unified picture, distilling the key ideas from a welter of sources. I have tried to give references to relevant original work, and can only apologise for any failures, which will have been unintentional.

I am very grateful for many conversations about supermanifolds that I have enjoyed and benefited from over the years - these have been with almost everyone whose work is referred to in this book, a sad exception being Berezin. I would particularly like to thank Bryce DeWitt and Marjorie Batchelor, both of whom have patiently explained many things to me. I am also grateful to my colleagues in the mathematics department of King's College London for a stimulating and agreeable working environment.

F. A. Rogers

King's College London 


\section{Contents}

Preface vii

1. Introduction 1

2. Super algebras $\quad 7$

2.1 The definition of a super algebra . . . . . . . . 7

2.2 Homomorphisms and modules of super algebras . . . . . 9

2.3 Super matrices . . . . . . . . . . . . . . . . . . 12

2.4 Super Lie algebras and super Lie modules . . . . . . . . . 13

$\begin{array}{ll}3 . & \text { Superspace } \\ \end{array}$

3.1 Real Grassmann algebras . . . . . . . . . . . . . . . . 17

3.2 The topology of superspace . . . . . . . . . . . . 21

3.3 Complex Grassmann algebras . . . . . . . . . . . . 23

3.4 Further super matrices . . . . . . . . . . . . . 25

4. Functions of anticommuting variables 31

4.1 Superdifferentiation and finite-dimensional Grassmann algebras ........................ 33

4.2 Taylor expansion and Grassmann analytic continuation . . 35

4.3 Supersmooth functions on $\mathbb{R}_{S}^{m, n} \ldots \ldots \ldots \ldots \ldots$

4.4 Properties of supersmooth functions . . . . . . . . . 40

4.5 Other infinite-dimensional algebras . . . . . . . . . 44

4.6 Obtaining well defined odd derivatives with finitedimensional Grassmann algebras . . . . . . . . . . . . 45

4.7 The inverse function theorem . . . . . . . . . . . 47 
4.8 Partitions of unity . . . . . . . . . . . . . . . 49

4.9 Superholomorphic functions of complex Grassmann variables 50

5. Supermanifolds: The concrete approach 51

$5.1 \quad G^{\infty}$ DeWitt supermanifolds . . . . . . . . . . . . . . . 52

5.2 The topology of supermanifolds . . . . . . . . . 55

5.3 More general supermanifolds . . . . . . . . . . . . . 56

5.4 The body of a supermanifold . . . . . . . . . . . . . 59

5.5 Complex supermanifolds . . . . . . . . . . . . . 61

6. Functions and vector fields 63

$6.1 G^{\infty}$ functions on supermanifolds . . . . . . . . . . . 64

6.2 Functions between supermanifolds . . . . . . . . . 67

6.3 Tangent vectors . . . . . . . . . . . . . . . . . 69

6.4 Vector fields . . . . . . . . . . . . . . . . . 74

6.5 Induced maps and integral curves . . . . . . . . . 78

7. Supermanifolds: The algebro-geometric approach 85

7.1 Algebro-geometric supermanifolds . . . . . . . . . . 85

7.2 Local coordinates on algebro-geometric supermanifolds . . 87

7.3 Maps between algebro-geometric supermanifolds . . . . . 89

8. The structure of supermanifolds 91

8.1 The construction of a split supermanifold from a vector bundle 92

8.2 Batchelor's structure theorem for $\left(\mathbb{R}_{S}^{m, n}\right.$, DeWitt, $\left.G^{\infty}\right)$ supermanifolds . . . . . . . . . . . . . . . . 94

8.3 A non-split complex supermanifold . . . . . . . . . . 96

8.4 Comparison of the algebro-geometric and concrete

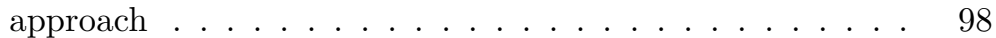

9. Super Lie groups 101

9.1 The definition of a super Lie group _ . . . . . . . . . 102

9.2 Examples of super Lie groups . . . . . . . . . . . . . . 104

9.3 The construction of a super Lie group with given super Lie

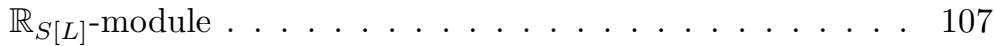

9.4 The super Lie groups which correspond to a given super Lie algebra . . . . . . . . . . . . . . . . . . 114 
9.5 Super Lie groups and the algebro-geometric approach to supermanifolds . . . . . . . . . . . . . . . 118

9.6 Super Lie group actions and the exponential map . . . . . 121

10. Tensors and forms 125

10.1 Tensors ....................... . . 125

10.2 Berezinian densities . . . . . . . . . . . . . . . 126

10.3 Exterior differential forms . . . . . . . . . . . . . . 127

10.4 Super forms . . . . . . . . . . . . . . . . . 131

11. Integration on supermanifolds 135

11.1 Integration with respect to anti commuting variables . . . 136

11.2 Integration on $\mathbb{R}_{S}^{m, n}$. . . . . . . . . . . . . . . . . . . . . 140

11.3 Integration on compact supermanifolds . . . . . . . . . . . 144

11.4 Rothstein's theory of integration on non-compact supermanifolds . . . . . . . . . . . . . . . . 146

11.5 Voronov's theory of integration of super forms . . . . . . . 152

11.6 Integration on $(1,1)$-dimensional supermanifolds . . . . . . 154

11.7 Integration of exterior forms . . . . . . . . . . . . 155

12. Geometric structures on supermanifolds 157

12.1 Fibre bundles . . . . . . . . . . . . . . . . . . 157

12.2 The frame bundle and tensors . . . . . . . . . . . . 160

12.3 Riemannian structures . . . . . . . . . . . . . . . . 161

12.4 Even symplectic structures . . . . . . . . . . . . . . . 162

12.5 Odd symplectic structures . . . . . . . . . . . . 164

13. Supermanifolds and supersymmetric theories 167

13.1 Superfields and the superspace formalism . . . . . . . . . . 170

13.2 Supergravity . . . . . . . . . . . . . . . . 175

13.3 Super embeddings . . . . . . . . . . . . . . . . . . . . 178

14. Super Riemann surfaces 181

14.1 The superspace geometry of the spinning string . . . . . . 182

14.2 The definition of a super Riemann surface . . . . . . . . . . 184

14.3 The supermoduli space of super Riemann surfaces . . . . . 186

14.4 Contour integration on super Riemann surfaces . . . . . . . 189

14.5 Fields on super Riemann surfaces . . . . . . . . . . . . . . 191 
15. Path integrals on supermanifolds 195

15.1 Path integrals and fermions . . . . . . . . . . . . . 195

15.2 Fermionic Brownian motion . . . . . . . . . . . . . 197

15.3 Brownian motion in superspace . . . . . . . . . . . . . . 199

15.4 Stochastic calculus in superspace . . . . . . . . . . . . 201

15.5 Brownian paths on supermanifolds . . . . . . . . . 203

16. Supermanifolds and BRST quantization 207

16.1 Symplectic reduction . . . . . . . . . . . . . 208

16.2 BRST cohomology . . . . . . . . . . . . . . . 211

16.3 BRST quantization . . . . . . . . . . . . . . . . 213

16.4 A topological example . . . . . . . . . . . . 215

17. Supermanifolds and geometry 221

17.1 Supermanifolds and differential forms . . . . . . . . . 222

17.2 Supermanifolds and spinors . . . . . . . . . . . . . 224

17.3 Supersymmetric quantum mechanics and the Atiyah Singer Index theorem . . . . . . . . . . . . . . 226

17.4 Further applications of supermanifolds . . . . . . . 233

Appendix A. Notation 237

$\begin{array}{ll}\text { Bibliography } & 239\end{array}$

$\begin{array}{ll}\text { Index } & 249\end{array}$ 